\title{
ANALYSIS OF DIFFERENT EDGE DETECTIONS ALGORITHMS THROUGH THE BIT-PLANE LAYERS
}

\author{
Ratko Ivković1, \\ Mile Petrović ${ }^{2}$ \\ Ivana Milošević ${ }^{3}$, \\ Branimir Jakšić ${ }^{2}$ \\ Risto Bojović ${ }^{2}$
}

${ }^{1}$ Faculty of Information Technologies, Alfa BK University,

Palmira Toljatija 3,

Belgrade, Serbia

${ }^{2}$ Faculty of Technical Sciencies,

University of Pristina,

Kneza Miloša 7 ,

Kosovska Mitrovica, Serbia

${ }^{3}$ SP Audio and Video Technologies,

The School of Electrical and

Computer Engineering,

Vojvode Stepe 283,

Belgrade, Serbia

\begin{abstract}
:
The research paper below gives analyses of different kinds of edge detection algorithms, with gradient structure, observed on a bit-plane model. Even though each bit contains certain part of information of the image, different algorithms for edge detection of the image do not perform identically, which is proved on a practical example of this work. Research has been conducted via the analysis done on the individual level through the exact image, as well as through the extent of 350 images characteristic for digital transformation of image. Each result, based on structural similarity model (SSIM), is given using the extent of the details level that was observed.
\end{abstract}

\section{Keywords:}

digital image processing, Bit-plane, edge detection algorithm, level of detail.

\section{INTRODUCTION}

Every bit of image memory contains certain information about the image. On the other hand, detection algorithms at different conditions give different results, which is proved in work papers [1] - [3]. Although according to the classic definition of digital transformation of image specified in literature [4], and many other literature sources considered to be fundamental in this field [5] - [7], higher bits are used for edge defining, but the relation between edge detection and bit plane is not explained. Based on quantitative research for each bit separately researchers gave suggestions and propositions that certain algorithm should be used for certain scientific domain, for example Sober for Robotics, etc.

It is important to point out that certain scientific fields that are highly dependent on transformation of large number of images, such as Photogrammetry or Forensics takes into consideration all kinds of bits of one image (all 8), [8]. Then, certain algorithms are applied to bring out confident conclusions. Processing time is decreased up to four times by reduction of processed bits. The above-mentioned fields use large bases of processed, that is compared, images for the analysis, which complicates the processes. If it were possible to approximately determine which bit contains most information regarding specific algorithm, then only some parts of image bits would be processed/compared.

These particularities of different edge detection algorithms have defined them for diverse purposes areas. Theoretically, advantages and

\section{Correspondence:}

Ratko Ivković

e-mail:

ratko.ivkovic@pr.ac.rs 
disadvantages could be discussed unlike superiority of an algorithm, neither in spatial domain nor in frequency domain.

\section{METHODOLOGY}

Original images were processed by an operator for digital images edge detection by decomposing them at the bit level by the bit 24 model record ( 8 bits per channel). Then each bit was compared with the image obtained with the help of a processing operator for edge detection with structural similarity model SSIM. All images used in processing were in TIFF format to avoid the possibility of losing them to the conversion. Also, all images were used in the theoretical framework of digital image processing and deliberations in various scientific works, so, the results of this work are measurable with other works. Representative image and part of the overall database of used images are shown in Fig. 1.

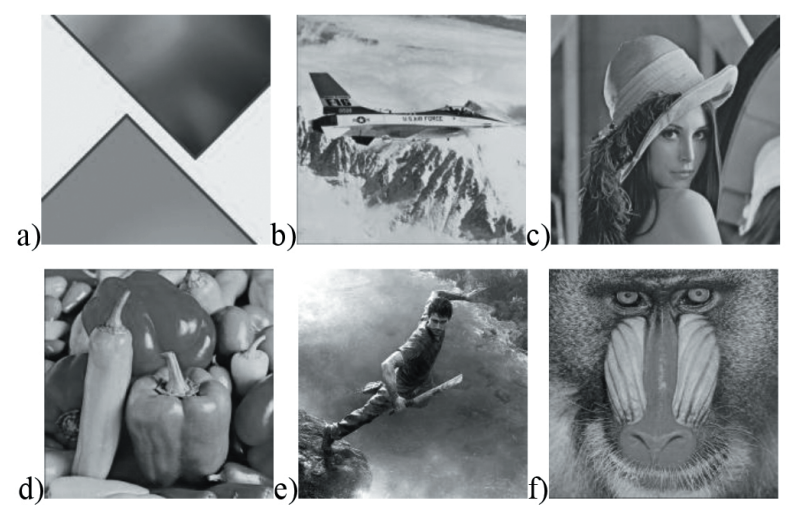

Fig. 1. Original images.

Used analyses included standard methods of theoretical bases for digital image processing. The level of details as a measure of change of some image parameter on observed part was a respectable parameter for image division into different categories for analysis. Range of processed images included complete characteristic range of level of details, divided into domains:

- Extremely Low Level of Detail (0 - 1.5),

- Low Level of Detail (1.51 - 3),

- Medium Level of Detail (3 - 5),

- High Level of Detail (6 - 7),

- Extremely High Level of Detail (7 and more).

\begin{tabular}{cc}
\hline Image & LoD (DCTD) \\
\hline Polygon & 0.16 \\
\hline Airplane & 1.606 \\
\hline Lena & 2.3296 \\
\hline Vegetable & 3,0335 \\
\hline Far Cry & 6.3703 \\
\hline Monkey & 7.9774 \\
\hline
\end{tabular}

Table 1. Level Of Detail for Original Images.

Analysis goal was to bring out joint conclusions via two completely separated analyses - an analysis through the separate examples and through the complete overview on all characteristic images.

The first analyzed specific range of level of details presented with one image that exemplified the certain range. This kind of process was repeated over and over for different edge detection algorithms with an aim, to determine the potential for each bit in regard to the assigned algorithm image edges.

The second one analyzed all images that included into the specific range of level of details. Results of this analysis would give compared review of algorithm for edge detection and their nature over bit plane.

\section{THEORETICAL BACKGROUND}

\section{Bit-plane}

If it is assumed that each pixel in image is presented by 8 bits. Those 8 bits could be decomposed into 8 layers. Figure 2 shows range from the layer 1 which contains bits with the lowest importance to the layer 8 containing bits with the highest importance. This implies when image is firstly converted into greyscale palette regarding lightening and reflections [9].

This kind of analysis is important because it gives relative importance for each bit of image. It determines number of bit which is used for each quantization pixel, and have an important role in image compression [10]. 
PIXEI

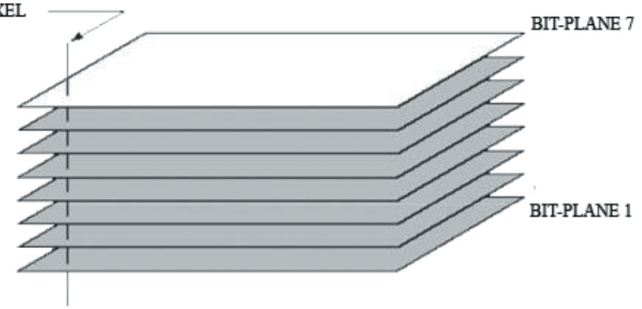

Fig. 2. Structure of image bit-plane.

\section{Level of Details (LoD)}

Level of details is a measure of pixel value variation on the observed image. Frequent changes are expressed as high level of details, and vice versa. This image phenomenon, in most cases, does not appear on complete observed image but only on certain parts of the image. Therefore, the level of details could be observed over spatial and frequency domain.

The simplest kind of edge detection is the one that appears when image is divided into rectangle $K x M$ pixels. This exact rectangle is used to measure number of pixels, number of changes or number of edges depending on defined ledge, inside of this rectangle. Formally and mathematically it is represented as follows:

$$
\delta_{i}=\frac{\delta_{n_{i}}}{k m} .
$$

Where $\delta_{n_{i}}$ relates to number of detected pixel edges inside of that rectangle $\mathrm{km}$ dimensions. In this case edge detection must be carried out over some algorithm used for edge detection. In most cases the used operator is a Laplacian operator for edge detection. As an outcome, errors in account can be counted based on errors defined by Laplacian operator. As well as range of observed pixels on which edges are defined in range between 0 and 1 , for some certain rectangle $\delta_{n_{i}}$ [1].

Next step depends on applied transformation over observed image.

\section{The structural similarity index (SSIM)}

To avoid subjective evaluation a procedures automated quality assessment is required. Automatic assessment procedure is regarded as an objective evaluation and is useful in many applications where it is necessary to evaluate the visual effects that occur in images during acquisition, processing, transmission, compression and archiving. With a software implementation, time of the assessment is reduced from days and weeks to a few seconds.
The main objective of automatic assessment of the quality of pictures/videos is to obtain predictions that are in good correlation with the average subjective assessment (MOS - Mean Opinion scope). The ideal objective measurement of quality assessment should be applicable in various types of distortion that quantitatively include varying degrees of distortion and consider the distribution of errors that occur. In practical applications in addition to the above-mentioned conditions computational complexity is also important $[11,12]$. The index describes the structural similarity of image quality by comparing local correlation of luminance, contrast, and structure between the source and test images.

\section{RESULTS \& DISCUSSION}

The results of the first analysis or comparative analysis of the edges detection algorithms seen through the prism of the band of detail level is given in Figures 3, 4, 5, 6, 7 .

The behavior of different edge detecting algorithms in an extremely low level of detail is shown in Fig. 1. As can be appreciated by almost all algorithms the highest level of information about similarities is in the eighth bit, except for Prewit algorithm. Also, it may be concluded that in the detection of the edges, Sobel and Canny operators are required to be taken into account in the 2, 3, 4, 5, 6 and 8 bit, because there is $98 \%$ of the amount of edge information. Laplacian and Roberts operator in terms of the edges are defined in 5, 6 and 8 bit.

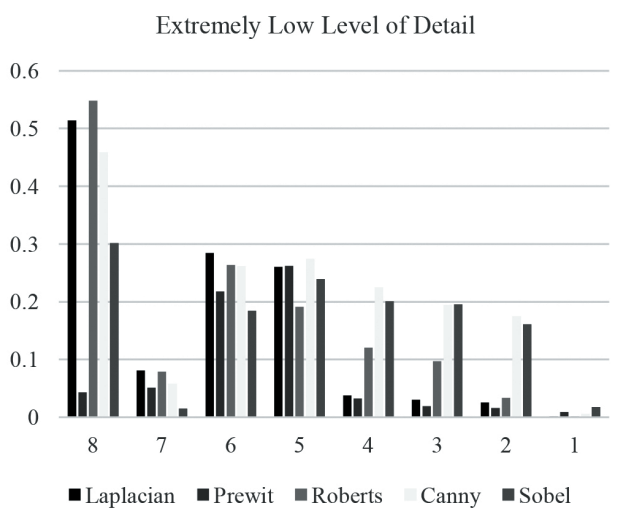

Fig. 3. Edge content in every bit of image, for various Edge Detection Algoritham trough Extremly Low Level of Detail.

Fig. 3 provides a comparative overview of algorithms for Low Level of Detail. It is very important to note that for Sobel operator of bit levels 5, 6, 7, and define more 
than $90 \%$ of the useful information, while for all other algorithms, it is necessary to observe from 5 to 8 bits.

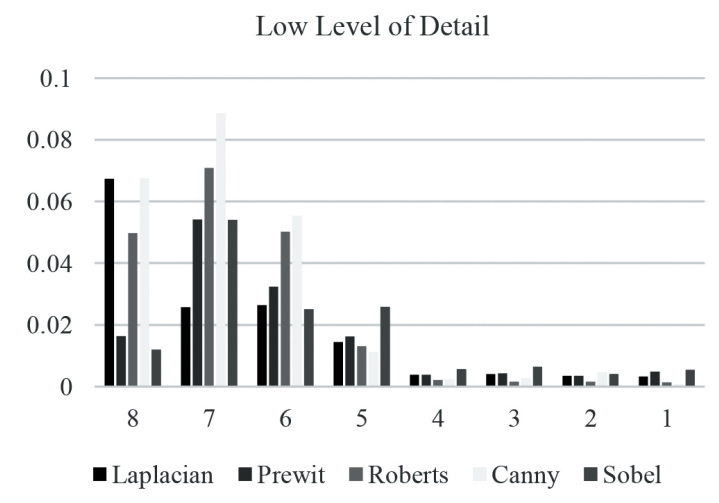

Fig. 4. Edge content in every bit of image, for various Edge Detection Algoritham trough Low Level of Detail.

For a medium level of detail given in Fig. 4, all algorithms can be defined with more than $85 \%$ of the information on edges in bit-planes 6, 7 and 8, while th e Laplacian can be defined through 6 and 8 bit. This information can significantly reduce processing time/comparisons in the use of algorithms to detect edges in the middle level of detail. Appraisal, that two or three bits carry a much larger part of the information about the edges in comparison with other bits, can affect the optimization of some image processing system.

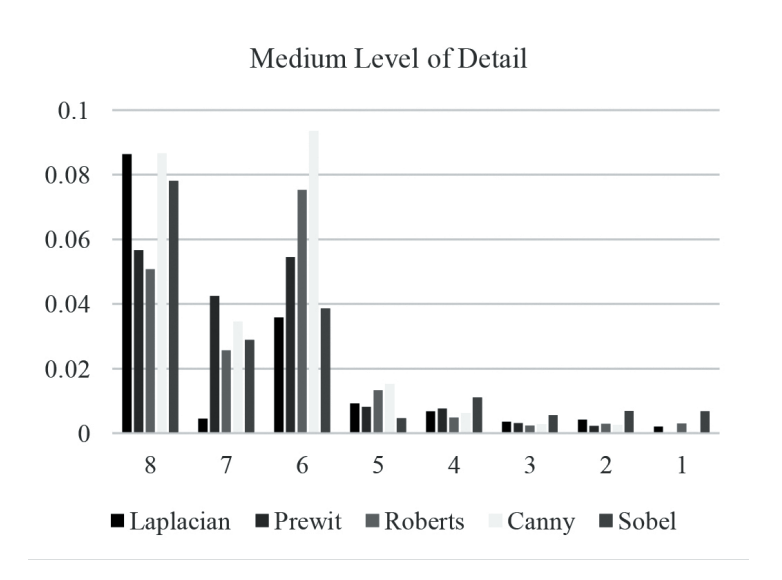

Fig. 5. Edge content in every bit of image, for various Edge Detection Algoritham trough Mediun Level of Detail.

The high level of detail given in Fig. 5 speaks of the high degree of concentration of information about the edges in 6,7 and 8 bit. It is necessary, in this area to pay attention to the Canny operator and concentration of information in 8 bit because there is $56 \%$ of the information. On the other hand, for Sobel operator in this case is necessary to take in the full extent because $32 \%$ of information is within the first 5 bits.

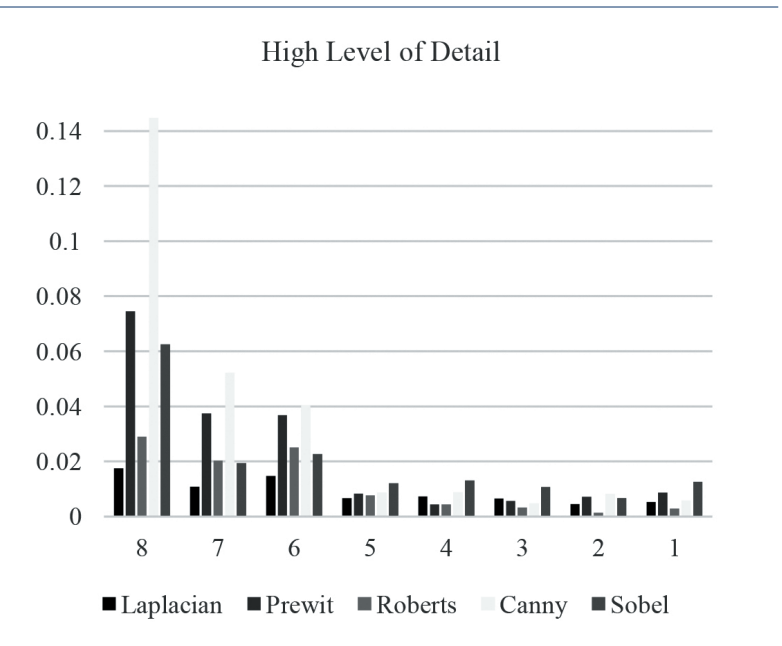

Fig. 6. Edge content in every bit of image, for various Edge Detection Algoritham trough High Level of Detail.

In order to avoid borderline cases, extremely low and extremely high level detail areas are separately evaluated. When it comes to extra high level of detail Laplacian, Prewit and Roberts is possible to observe the last three bits, while other operators should be treated based on all eight bits. This extreme situation suggests the difference in detection mode for images with extreme level of details.

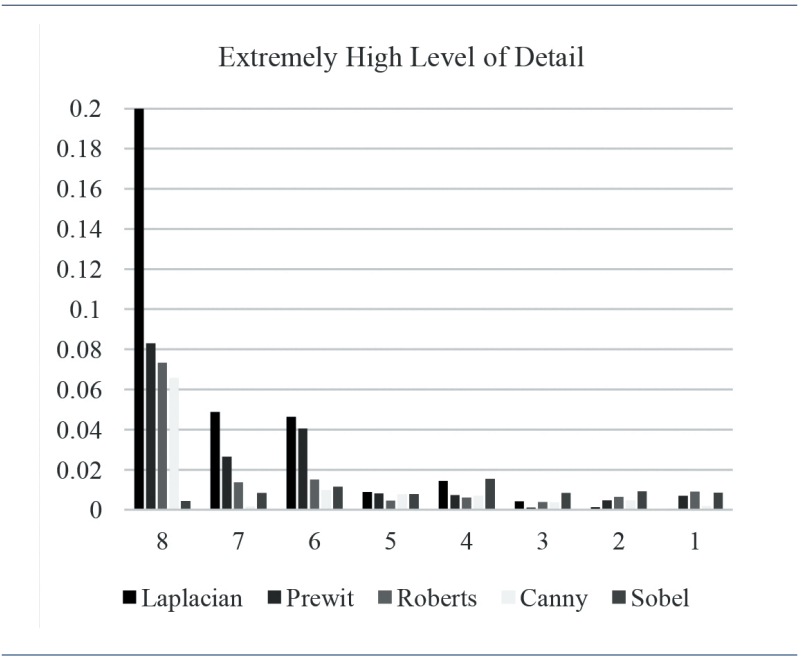

Fig. 7. Edge content in every bit of image, for various Edge Detection Algoritham trough Extremely High Level of Detail. 
The second segment of analysis included an analysis of each operator individually for the characteristic image for each band level of detail. In this analysis, it can be more clearly determined, the concentration ratio of edges information for each bit of the image. As you can notice the Laplacian operator for edge detection, $78 \%$ of the information of the image edges are in the 5, 6 and 8 bit. Regarding this, it may be proposed for future tests, that in these situations when using Laplacian operator only three bits mentioned are used, with the evaluation of detection/recognition of $78 \%$.

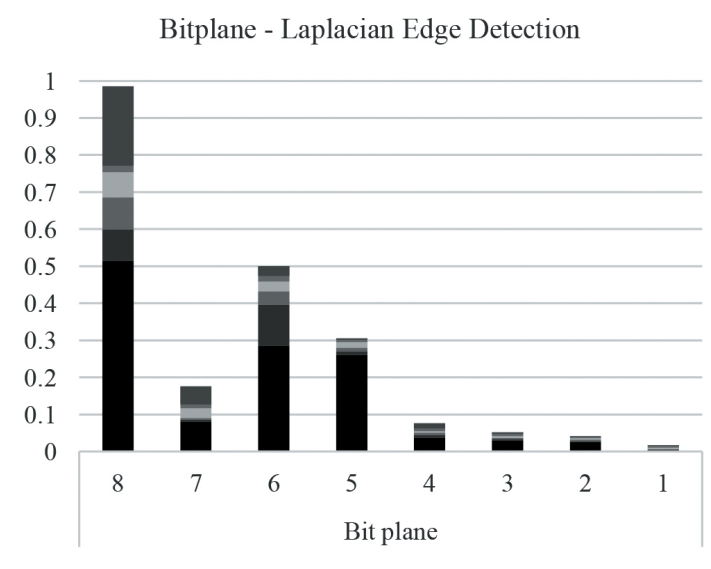

Fig. 8. Laplacian Edge Detection.

Prewit edge detection operator can boast only 7.5\% of the amount of information in the first 4 bits of the image. Which represents clearer situation as opposed to the Laplacian operator. The percentage of $92.5 \%$ in the last four bits actually gives in use processing/comparisons used the last 4 bits of the image. Although distinctive representative of $2 \mathrm{D}$ gradient operators, Prewit is precisely on this characteristic different from other operators.

Roberts operator suggests a bit different situation. In fact, $55 \%$ of complete information about the edges are 6 and 8 bit bit-level. While along with bits 4,5 and 7 , it is higher than $90 \%$.

Canny operator for detection of edge as one of the derivatives of Sobel operator, provides insight into the analysis of complete spectrum of the level of detail in Fig. 9. The first to notice is ratio between these operators and low and extremely low level of detail. In the extremely low level of detail, almost all bits must be considered for the purpose of an analysis/comparison. Whereas, for a low level of detail only 7 and 8 bit are considered. Other values are far lower, and for image analysis with this operator all bits must be taken into account.

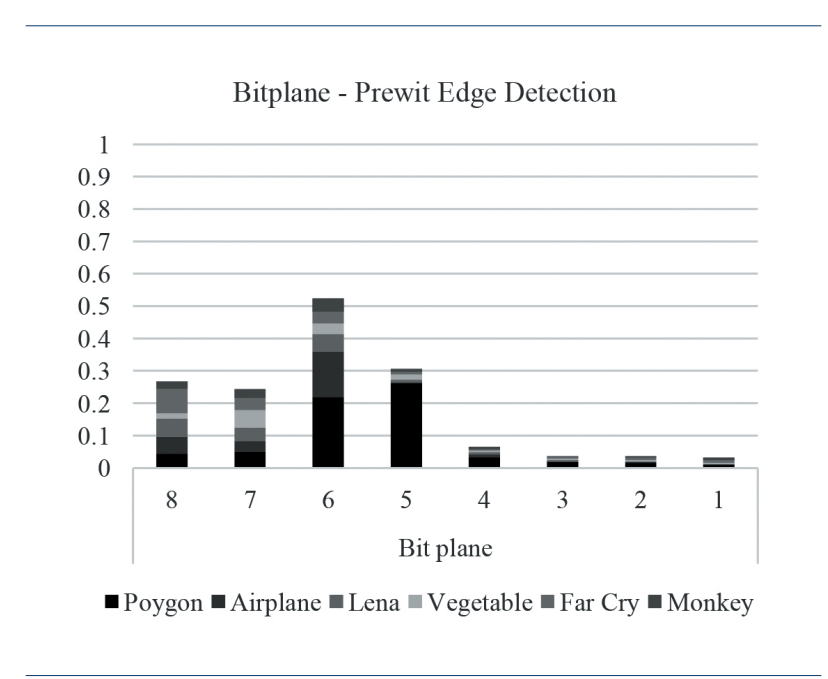

Fig. 9. Prewit Edge Detection

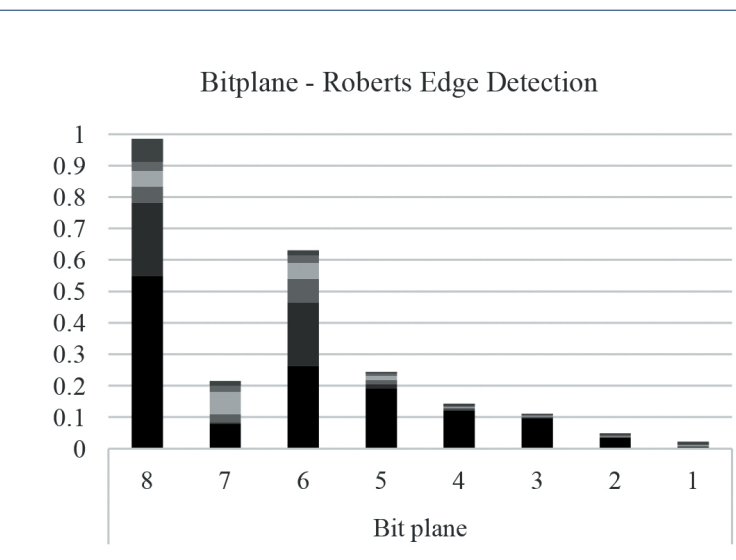

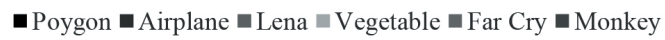

Fig. 10. Roberts Edge Detection.

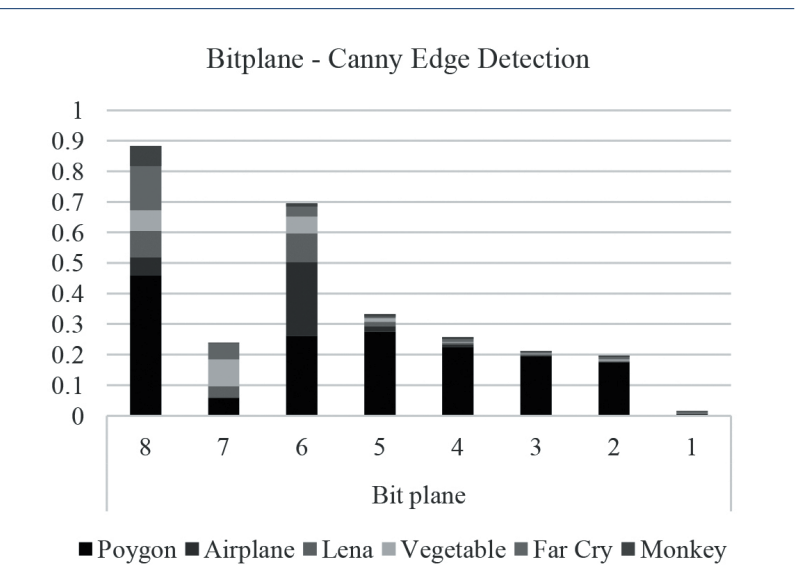

Fig. 11. Canny Edge Detection.

Sobel edge detection operator as one of the most often cited in research papers is given in Fig. 12. Viewed through all image characteristic of the complete spectrum of level of detail, it can be clearly concluded that nearly 
all bits must be processed to get the desired information. In this case, the smallest exception can be made, and in potential work with bits with Sobel detection optionally 1 and in some cases 7 bit may be neglected. These individual cases are related to high and extra high level of detail.

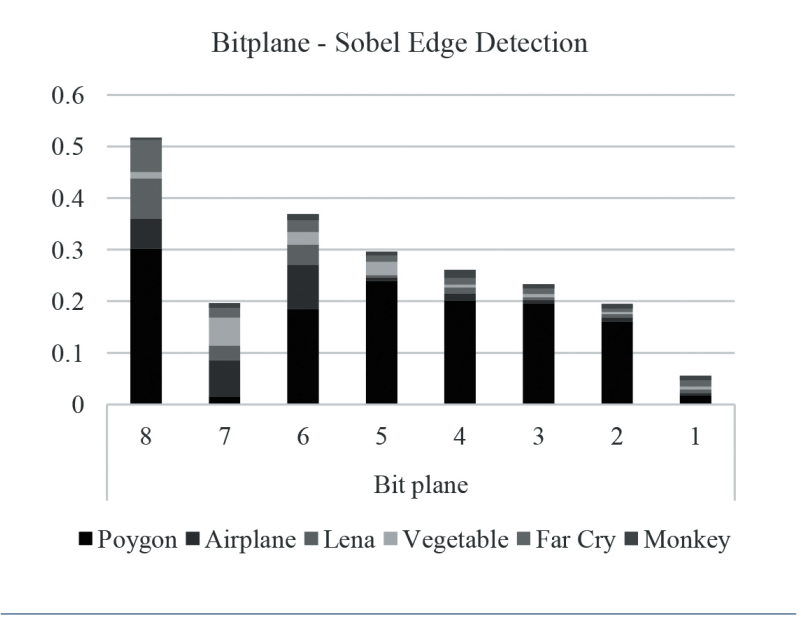

Fig. 12. Canny Edge Detection.

\section{CONCLUSION}

Present research work provided the analysis of different types of digital images edge detection operators, through a complete spectrum of level of detail divided into certain categories. The analysis unambiguously showed some significant results that can be used in the optimization of certain systems that use a base with a large number of images. Future research will move toward possibilities that images used in forensics or in photogrammetry be optimized for operation in the existing system thus reducing processing time.

\section{ACKNOWLEDGMENT}

This work was done within the research project of the Ministry of Science and Technological Development of Serbia TR35026, III47016 and III44006.

\section{REFERENCES}

[1] R. Ivkovic, I. Milosevic, B. Gara, M. Pavlovic and D. Miljkovic, "Analysis of Digital Image Segments Through the Standard Deviation and Level of Detail", INFOTEH 2015, Jahorina, Bosna i Hercegovina, Vol. 14, pp. 600-603, March 2015.

[2] R. Ivkovic, I. Miloševic, B. Gara, S. Minic and V. Dakovic, "Algoritam za detekciju ivica slike sa postfiltrom", INFOTEH 2014, Jahorina, Bosna i Hercegovina, Vol. 13, pp. 652-656, 19-21 Mart 2014.

[3] B. Jaksic, R. Ivkovic, B. Gara, M. Petrovic and P. Spalevic, "Analysis of different influence of compression algorithm on the image filtered Laplcian, Prewitt and Sobel operator", International Journal of Darshan Institute on Engineering Research and Emerging Technology, vol. 2, no. 1, pp. 59-67, 2013.

[4] R.1 C. Gonzalez and R. E. Woods, "Digital Image Processing”, vol. III, Pearson, England, 2007, pp. 57243.

[5] T. Young, J. J. Gerbrands and L. J. van Vliet, "Fundamentals of Image Processing", Delft University of Technology Press, 2007, pp. 36-75.

[6] I. Pitas, "Digital Image Processing Algorithms", Prentice Hall, Englewood Cliffs, NJ, 1993, pp. 169193.

[7] E. R. Dougherty, "Random Processes for Image and Signal Processing", New York:Wiley-IEEE Press, 1998, pp. 135-172.

[8] I. Milosevic, R. Ivkovic, T. Petrovic, N. Denic and B. Jaksic, "Face Recognition through Robust Multimodal Biometric System with Sobel Edge Detection", International Scientific Conference "UNITECH 2015”, Gabrovo, Bulgaria, 20-21, November 2015, Proceedings, Vol. 2, pp. II346-II351.

[9] E.R. Dougherty, "Random Processes for Image and Signal Processing", IEEE Press, 2000, pp. 43-112.

[10] R.C.Gonzales, R.E.Woods, "Digital Image Processing", 2th Edition, Prentice Hall, 2007, pp. 98-123.

[11] Z. Wang, A.C. Bovik and L. Lu, "Why is Image Quality Assessment So Difficult?", Proceedings of ICASSP, May 2002.

[12] A. Shnayderman, A. Gusev and A. M. Eskicioglu, "An SVD-Based Gray-Scale Image Quality Measure for Local and Global Assessment", IEEE Trans. on Image Proc., Vol. 15, No. 2, 2006, pp. 422-429. 